\title{
Introduction : génétique et théâtre
}

\section{Françoise Simonet-Tenant}

URL : http://journals.openedition.org/coma/688

DOI : $10.4000 /$ coma.688

ISSN : 2275-1742

\section{Éditeur}

Institut des textes \& manuscrits modernes (ITEM)

\section{Référence électronique}

Françoise Simonet-Tenant, «Introduction : génétique et théâtre », Continents manuscrits [En ligne]

7 | 2016, mis en ligne le 15 décembre 2016, consulté le 01 mai 2019. URL : http:// journals.openedition.org/coma/688; DOI : 10.4000/coma.688

Ce document a été généré automatiquement le 1 mai 2019.

\section{(c) $(1) \Theta \Theta$}

Continents manuscrits - Génétique des textes littéraires - Afrique, Caraîbe, dispora est mis à disposition selon les termes de la licence Creative Commons Attribution - Pas d'Utilisation Commerciale - Pas de Modification 4.0 International. 


\title{
Introduction : génétique et théâtre
}

\author{
Françoise Simonet-Tenant
}

1 L'objet de la critique génétique : « les manuscrits littéraires en tant qu'ils portent la trace d'une dynamique, celle du texte en devenir. Sa méthode: la mise à nu du corps et du cours de l'écriture, assortie de la construction d'une série d'hypothèses sur les opérations scripturales. Sa visée: la littérature comme un faire, comme une activité, comme mouvement ${ }^{1}$.» Telle est la définition donnée par Almuth Grésillon dans un ouvrage fondateur, Éléments de critique génétique. Autrement dit, il s'agit de remonter le cours de l'œuvre jusqu'à sa source, de scruter inlassablement les brouillons (notes préparatoires, esquisses, premiers états d'un texte) pour reconstituer l'histoire de la vie d'un texte. Curiosité dévoyée et stérile pour certains, la crique génétique est, pour d'autres, une recherche austère qui vise une connaissance raisonnée des actes de la créativité verbale. Cette exigence de rigueur scientifique n'exclut pas une part de fièvre de la part d'un critique qui se fait limier, ni une part de rêve - rêve d'intimité du critique qui s'introduit par effraction dans les arcanes de la création et quête passionnée dont le Graal serait l'inobservable : l'origine même de l'œuvre littéraire.

2 C'est dans les années 1970 que se mettent en place en France les conditions d'une nouvelle aventure critique. Louis Hay fonde en 1974 le Centre d'analyse des manuscrits du CNRS, institut rebaptisé ITEM en 1982 (Institut des Textes et Manuscrits Modernes). Les premiers chantiers génétiques se concentrent sur quelques grands corpus littéraires: Heine, Nietzsche, Flaubert, Zola, Proust, Valéry, Joyce, Aragon... Au fil des années l'objet de la critique génétique se diversifie, s'ouvrant aux écritures autobiographiques, aux écritures scientifiques, aux arts plastiques, au cinéma, à la musique, et maintenant aux écritures francophones. L'attention portée à la genèse théâtrale commence dans les années 1990. L'on peut en noter les jalons essentiels : le séminaire général de l'ITEM est consacré de 2004 à 2006 aux "genèses théâtrales »; le numéro 26 de Genesis est consacré en 2005 au "Théâtre »; une entrée "Critique génétique » est ajoutée dans le Dictionnaire encyclopédique du théâtre lors d'une réédition en $2008^{2}$; Almuth Grésillon, Marie-Madeleine Mervant-Roux et Dominique Budor publient en 2010 un ouvrage collectif intitulé Genèses théâtrales (CNRS Editions) qui prolonge et approfondit les perspectives ouvertes par le numéro 26 de la revue Genesis. Nous n'oublierons pas non plus les recherches menées au 
Canada et dans le monde anglophone : les travaux, entre autres, d'Irène Roy et de Josette Féral ${ }^{3}$. Cette dossier s'inscrit donc sur un arrière-plan critique déjà peuplé mais où il reste encore beaucoup à faire.

3 L'étude des genèses théâtrales a en effet mis à l'épreuve la pensée généticienne et a mis au jour de nouvelles questions et problématiques articulées autour d'un questionnement central : «Est-il possible de reconstruire le processus complexe qui préside à la genèse d'une œuvre théâtrale ${ }^{4}$ ?»

4 Première question: sans tomber dans une dichotomie artificielle et dans le «binôme historiquement daté du texte et de la scène ${ }^{5}$ ", il est nécessaire de prendre en compte à la fois la genèse du texte et la genèse de la scène dont la relation d'interdépendance donne à la genèse théâtrale sa spécificité et son dynamisme. L'on sait que «l'expérience concrète de la mise en scène peut provoquer en retour des réécritures du texte, voire, comme on l'observe notamment dans le théâtre contemporain, la fixation définitive de la matière textuelle elle-même ${ }^{6}$.» Une simple preuve lexicale en est l'existence même du terme de becquet, qui désigne le fragment de texte ajouté par l'auteur au cours des répétitions. L'on sait par exemple combien la pièce de Jean Giraudoux, Intermezzo, se transforma entre mars 1931, date de son achèvement provisoire, et le $1^{\mathrm{er}}$ mars 1933, date de la création de la pièce. Entre temps, sous l'influence notable de Jouvet, le metteur en scène, les feuillets de la pièce se truffèrent de becquets et variantes et comptèrent nombre de coupures ${ }^{7}$. Ainsi le nombre des témoins scripturaires de la genèse théâtrale, lorsqu'ils ont été conservés, peut être élevé : l'on citera au moins, sans préjuger de leur existence dans tous les cas de genèse, les avant-textes du texte dialogué et du texte didascalique, les notes préparatoires du metteur en scène, les écritures de régie, les brochures d'acteurs, le plan ou schéma d'implantation, les croquis de costumes, les plans de décors, la note d'interprétation inscrite par le metteur en scène dans le programme, les manuscrits du souffleur, les journaux de travail éventuellement tenus par certains des différents intervenants du spectacle (à cet égard on peut donner pour exemple les journaux de JeanFrançois Peyret, 2001 à 2008, mis en ligne). À cette liste déjà longue on pourrait ajouter les pièces juridiques et financières qui accompagnent la fabrique du spectacle, voire des témoins non scripturaires tels que les photographies ou les captations de répétitions. Cette abondance possible peut évidemment donner le vertige au critique d'autant plus que se pose alors une difficulté notable. Ce qui fait la spécificité de la génétique, c'est la dimension diachronique de l'étude. Dans le cas de la genèse théâtrale caractérisée par l'élaboration double entre genèse textuelle et genèse $d u$ spectacle, pourra-t-on chronologiser les différentes traces laissées par le processus de production et pourra-t-on véritablement reconstituer le fil d'un processus?

Deuxième question : dans une genèse théâtrale, plus que dans une genèse romanesque ou poétique, s'impose une interrogation. Qui est l'auteur? Ni le texte de théâtre, ni le dramaturge - au sens d'auteur d'un texte destiné à la scène - ne sont plus, depuis longtemps, sacralisés, et les études théâtrales, discipline créée en France dans les années 1960, ont largement contribué à cette désacralisation. Le binôme d'auteurs - dramaturge et metteur en scène - a semblé un temps une hypothèse recevable, confortée par des tandems illustres : Giraudoux et Jouvet, Claudel et Jean-Louis Barrault, Ionesco et Jacques Mauclair, Cixous et Mnouchkine, Duras et Claude Régy, Koltès et Chéreau. Ce modèle semble contesté à l'heure où l'on introduit la notion "d'auteurs en scène ${ }^{8}$ » et où la hiérarchie des rôles dans la chaîne de la production théâtrale est bousculée. L'on peut prendre l'exemple de Joël Pommerat qui définit ainsi sa création : 
Je n'ai jamais pu donner à un scénographe un texte à lire et attendre qu'il me fasse des propositions. [...] La scénographie, c'est-à-dire l'espace dans lequel une fiction va pouvoir se déployer, appartient chez moi intégralement au domaine de l'écriture. Ce n'est pas annexe. L'espace de la représentation, celui dans lequel les figures ou personnages vont évoluer ou vivre, c'est la page blanche au commencement d'un projet. Depuis que j'ai commencé à faire des spectacles (au début des années 1990), je me suis toujours défini comme «écrivant des spectacles" et non pas comme "écrivant des textes ». En tant qu'écrivain de spectacles, j'ai toujours commencé par définir (et j'y tiens) pragmatiquement des grands principes de scénographie'. Il paraît donc parfois difficile de distinguer strictement les postes de travail et, comme le remarquent à juste titre les auteurs de Genèses théâtrales, "le caractère interartistique de la scène contemporaine (mêlant danse, arts plastiques, cirque, théâtre d'objets...) accentue la démultiplication des pôles créatifs ${ }^{10} »$;

de là la difficulté d'individualiser les décisions. Le théâtre comme le cinéma - auquel s'est également attaquée la critique génétique - suscite un processus créatif où interviennent de nombreux collaborateurs qui réagissent l'un à l'autre.

Troisième question : comme nous l'avons suggéré au début de notre exposé, il s'agit en génétique de remonter le cours de l'œuvre jusqu'à son origine. Cette remontée labyrinthique s'adosse à l'état considéré comme définitif d'une œuvre. Or à quel moment peut-on considérer qu'une genèse théâtrale est achevée? L'œuvre théâtrale est-elle un jour fixée?

7 Théâtre et génétique se questionnent donc mutuellement: d'une part, "l'originalité du phénomène théâtral offre une occasion d'exercice à la pensée généticienne ${ }^{11}$ ", conduite à aborder sous un nouvel angle les questions de l'auteur, de l'inachèvement, de l'archivage ; d'autre part, la perspective génétique renouvelle l'approche critique du théâtre, démontrant l'interdépendance du texte et de la scène - dont la distinction n'est pas opératoire - et l'élaboré éphémère qu'est la genèse théâtrale. Les textes recueillis dans ce dossier permettront d'appliquer ces questionnements à des corpus de théâtre de langue française ancrés dans des aires géographiques différentes: Canada, Caraïbes, France, Maghreb. Espérons que ces corpus seront un excellent terrain d'interrogations et apporteront peut-être de nouvelles réponses.

\section{NOTES}

1. Almuth Grésillon, Eléments de critique génétique, Paris, PUF, 1994, p. 7.

2. Michel Corvin (dir.), Dictionnaire encyclopédique du théâtre à travers le monde, Paris, Bordas / SEJER, 2008 (1991). L'entrée « critique génétique » est rédigée par Jean-Yves Vialletton et JeanMarie Thomasseau.

3. Voir, entre autres, Josette Féral, Théorie et pratique du théâtre, Montpellier, Éditions de l'entretemps, 2011 et Irène Roy, "Cycles Repère : stratégie de création et genèse du spectacle ", dans Bernard Paquet (dir.), Faire œuvre. Transparence et opacité, Québec, Presses de l'université Laval, 2009, p. 157-166.

4. Almuth Grésillon, Marie-Madeleine Mervant-Roux, Dominique Budor, Genèses théâtrales, CNRS éditions, Paris, 2010, p. 8. 


\section{Ibid., p. 5.}

6. Almuth Grésillon et Jean-Marie Thomasseau, «Scènes de genèses théâtrales ", Genesis, $\mathrm{n}^{\circ}$ 26, 2005, p. 19.

7. Marie-Odile Germain et Danièle Thibault (dir.), Brouillons d'écrivains, Bibliothèque nationale de France, 2001, p. 151.

8. Voir Julie Sermont, Jean-Pierre Ryngaert, Théâtres $d u$ XXI ${ }^{e}$ siècle : commencements, Paris, Armand Colin, 2012, p. 52 : « Des auteurs écrivent désormais depuis le plateau, on peut donc les désigner littéralement comme des "auteurs en scène " calqué sur "metteur en scène“ (M. Cousin) ou comme des “écrivains de plateau” (B. Tackels). Ainsi, l'Argentin R. Garcia ou le Français J. Pommerat s'emparent simultanément des deux activités. "

9. Entretien de Joël Pommerat avec Christian Longchamp mis en ligne à l'adresse: http:// www.theatre-contemporain.net/spectacles/Cendrillon/ensavoirplus/

10. Almuth Grésillon, Marie-Madeleine Mervant-Roux, Dominique Budor, op. cit., p. 16.

11. « Théâtre (genèses théâtrales), Dictionnaire de critique génétique. http://ww.item.ens.fr

\section{RÉSUMÉS}

Après avoir rappelé les étapes qui ont permis au domaine de la "critique génétique » de se formuler, Françoise Simonet-Tenant récapitule ici les caractéristiques spécifiques de la genèse théâtrale. Le texte et la représentation s'organisent en effet souvent ensemble. Dès lors, le nombre d'intervenants dans l'élaboration de la pièce augmente et ne se cantonne plus seulement à « l'auteur ", et la chronologie préparatoire de la pièce se révèle plus difficile à tracer. Celle-ci semble être en fait toujours dans un état transitoire, à renouveler sans cesse au gré des représentations successives.

After having reminded the key stages of genetic criticism development, Françoise SimonetTenant, resumes specific features of theatrical genesis. Indeed, the text and the performance are often tightly linked one to another. Thus, there are more and more protagonists in piece's conception which is never limited to the "author"; and the piece's preparation chronology reveals difficult to map out. In fact, it seems always transient, in a constant renewal of following performances.

\section{INDEX}

Mots-clés : théàtre, genèse théâtrale, critique génétique, représentation

Keywords : theater, genesis of the theatrical writing, genetic criticism, performance

\section{AUTEUR}

\section{FRANÇOISE SIMONET-TENANT}

Université de Rouen - CEREdI 